\title{
Subtractive assembly for comparative metagenomics, and its application to type 2 diabetes metagenomes
}

\author{
Mingjie Wang ${ }^{1}$, Thomas G. Doak ${ }^{2,3}$ and Yuzhen Ye
}

\begin{abstract}
Comparative metagenomics remains challenging due to the size and complexity of metagenomic datasets. Here we introduce subtractive assembly, a de novo assembly approach for comparative metagenomics that directly assembles only the differential reads that distinguish between two groups of metagenomes. Using simulated datasets, we show it improves both the efficiency of the assembly and the assembly quality of the differential genomes and genes. Further, its application to type 2 diabetes (T2D) metagenomic datasets reveals clear signatures of the T2D gut microbiome, revealing new phylogenetic and functional features of the gut microbial communities associated with T2D.
\end{abstract}

Keywords: Comparative metagenomics, Subtractive assembly, Bloom filter, Type 2 diabetes

\section{Background}

Metagenomics relies on the direct sequencing of an entire community of microbial organisms, but the results can be hard to disentangle [1]. Microbial communities vary in compositional complexity [2], from the simplest acid mine drainage microbial community with a few species to more complex microbial communities that may contain hundreds - even thousands - of microbial species (such as the human microbiome [3]). Even though many new methods and tools have been developed for analyzing metagenomic sequences, it remains a great challenge to infer the composition and functional properties of a microbial community from a metagenomic dataset, and to address causal questions, such as the impact of microbes on human health and diseases. Metagenomic assembly (the assembly of metagenomic samples) is one of the challenges. While assembly of a single genome using short reads has improved in recent years, even that remains an area of active improvement [4]. In the case of metagenomic datasets, it is difficult for conventional genome assemblers to deal with closely related strains and to distinguish true variations from sequencing

\footnotetext{
* Correspondence: yye@indiana.edu

'School of Informatics and Computing, Indiana University, Bloomington, IN 47405, USA

Full list of author information is available at the end of the article
}

errors [5]: using simulated Illumina reads from a 400genome community, Mende et al. [6] found that relatively few of the reads were assembled, and of the contigs produced, $37 \%$ were chimeric. Also, the varied depth of coverage across the individual chromosomes leads to ambiguity in assembly [7]. Finally, the sheer size of metagenomic datasets poses a challenge, as sufficient sequencing must be done to represent ever rarer members of the community [7]. But there is much to be learned by comparing metagenomic datasets sampled from different environments (or hosts): metagenomics can be used to reveal important connections between microbes and other aspects of life (such as human health and disease). A recent exemplar is the identification of a connection between microbes and type II diabetes [8]. Comparative metagenomics studies how environment and/or health correlate with microbial communities phylogenetically and functionally, using either $16 \mathrm{~S}$ ribosomal RNA data or whole genome shotgun metagenomic sequence data [9]. Early studies compared the genomic diversity and metabolic capabilities across dramatically different metagenomes using barely assembled sequence data [10], while recent studies are more concerned with investigating how environmental or health features correlate with metagenomic differences using largely similar metagenomes [11-14]. 
Traditional comparative metagenomics begins with estimating biodiversity using short reads [15], or characterizing the biological and functional profiles based on known databases [16, 17]. Maillet et al. [18] proposed an approach that compares multiple metagenomic samples by efficiently identifying shared or similar reads based on $k$-mers and Jiang et al. [19] further developed several statistics that measure the dissimilarity between samples using sequence signatures (frequencies of $k$-mers) and applied them to metagenomics. It has also been reported that sequence signatures are similar for fragments from the same genome, but distinct between genomes [20]. Therefore, metagenomes with different microbial compositions tend to have distinctive sequence signatures, and the similarity and dissimilarity of metagenomes can be calculated using short reads without using any prior information.

Here we propose a subtractive assembly approach, a de novo method to compare metagenomes through metagenomic assemblies, aiming to achieve better assembly of the "differential" genomes for downstream analysis (e.g., to infer potential microbial markers associated with a human disease). For two or more metagenomes, reads that constitute the compositional difference are extracted from each metagenome based on sequence signatures. For example, we may define $k$-mers that occur ten times more frequently in one dataset than in the other as "signatures" that constitute the genomic difference; reads containing these signatures are likely to be from genomes that are more abundant or even unique in one of the two metagenomes. After read filtering, the complexity of the metagenome data sets can be greatly reduced, such that metagenome assembly using the extracted distinctive reads can be improved due to reduction in both biological diversity and data size. The compositional and functional difference of metagenomes can thus be characterized by the better-assembled contigs obtained from subtractive assembly.

For $k$-mer-based methods, a crucial step is the counting and storing of all $k$-mers. A number of efficient $k$ mer counting algorithms are publicly available [21-23]: BFCounter [22] adopts a bloom filter, making it quite memory efficient and thus most suitable for comparative metagenomics. We modified the $\mathrm{C}++$ code of BFCounter to output reads with distinctive signatures. With simulated metagenomic datasets, we show that subtractive assembly can both effectively extract the reads from genomes that cause the compositional differences between metagenomes and improve metagenomic assembly for these genomes.

Our subtractive assembly is superficially similar to the method developed by Stranneheim et al. [24], which reduces the complexity of the metagenome assembly problem by filtering out reads that can be classified to known genomes, assuming that they are often of no interest. Our subtractive assembly approach takes advantage of the availability of metagenomic datasets of the same community under different conditions: when we are interested mostly in the differences between two (groups of) metagenomes, we can assemble only the differences by filtering out reads that are likely to have been sampled from species that are common to both samples. Our method is independent of reference genomes.

Type 2 diabetes (T2D) is one of the many diseases that have an associated microbial "profile": it is associated with increased levels of streptococci, lactobacilli and Streptococcus mutans in oral samples [25]; Lactobacillus in gut microbiota is linked to obesity in humans, and weight gain for newborn ducks and chicks [26-28]; and Karlsson et al. [8] found that four Lactobacillus species and $S$. mutans are enriched in the gut microbiota of European women with T2D, using a large cohort of gut microbiome datasets. We applied our method to these gut metagenomes to see if our method could replicate the previous results, and perhaps further them: our subtractive assembly revealed new phylogenetic and functional features of the gut microbial communities associated with T2D.

\section{Results and discussion}

We first tested subtractive assembly using simulated metagenomic datasets, and then applied it to the datasets from [8], to identify differential features of the T2Dassociated microbiome. Our results show that the compositional difference of multiple metagenomic datasets could be detected using our $k$-mer-based method. Moreover, subtractive assembly utilizing only the reads that represent the compositional difference substantially reduced the complexity of the datasets and greatly improved the quality of the resulting assemblies, facilitating identifying compositional and functional differences between microbiomes. Application of our approach to the T2D datasets resulted in a large collection of genes that are uniquely found in the T2D-associated gut microbiomes, but which had not previously been identified.

\section{Evaluation of subtractive assembly: effectiveness of differential reads extraction and the requirement for abundance differences}

We first tested the effectiveness of the $k$-mer-countingbased extraction of differential reads, using simulated metagenomic samples composed of five bacterial genomes (in three groups of five, four and three samples; Table 1; real microbiomes, such as the gut microbiomes which we analyze below, can be much more complex). In each group, S1 has a uniquely large proportion of Streptococcus thermophilus reads. For each of the groups, sample 1 (S1) was subtracted by each of the other samples (S2, S3 and 
Table 1 Species composition of the artificial metagenomic samples in simulation 1

\begin{tabular}{|c|c|c|c|c|c|c|c|c|c|c|c|c|}
\hline & \multicolumn{5}{|c|}{ Group 1} & \multicolumn{4}{|c|}{ Group 2} & \multicolumn{3}{|c|}{ Group 3} \\
\hline & S1 & S2 & S3 & S4 & S5 & S1 & S2 & S3 & S4 & S1 & S2 & S3 \\
\hline Ferroplasma acidarmanus fer 1 & $1^{\mathrm{a}}$ & 16 & 1 & 1 & 1 & 1 & 12 & 1 & 1 & 1 & 10 & 1 \\
\hline Lactobacillus gasseri ATCC 33323 & 2 & 2 & 16 & 2 & 2 & 2 & 2 & 12 & 2 & 2 & 2 & 10 \\
\hline Pediococcus pentosaceus ATCC 25745 & 4 & 4 & 4 & 16 & 4 & 4 & 4 & 4 & 12 & 4 & 4 & 4 \\
\hline Prochlorococcus marinus NATL2A & 8 & 8 & 8 & 8 & 16 & 8 & 8 & 8 & 8 & 8 & 8 & 8 \\
\hline Streptococcus thermophilus LMD-9 & 16 & 1 & 2 & 4 & 8 & 12 & 1 & 2 & 4 & 10 & 1 & 2 \\
\hline RA ratio $\left(S_{1} / S_{i}, i !=1\right)^{\mathrm{b}}$ & & $16^{\mathrm{c}}$ & 8 & 4 & 2 & & 12 & 6 & 3 & & 10 & 5 \\
\hline
\end{tabular}

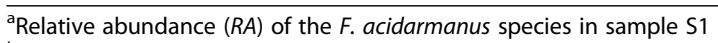

${ }^{\mathrm{b}}$ Relative abundance of the $\mathrm{S}$. thermophilus genome in $\mathrm{S} 1$ relative to $\mathrm{S} 2, \mathrm{~S} 3$ and so on. Pairs of datasets (S1 in each group, and another one in the same group) were subjected to subtractive assembly

'The relative abundance of the S. thermophilus genome in S1 versus S2

so forth) and the remaining reads were used for assembly. The fold change of the $S$. thermophilus genome ranges from 2-16 (Table 1). We examined how the assembly coverage of the $S$. thermophilus reference genome changes when the parameters, including the actual abundance ratio of the genome in two metagenomes (or the fold change) and the $k$-mer ratio threshold used in the subtractive assembly, are changed (Fig. 1; for real metagenomes, we used an iterative subtractive assembly approach without fixing the $k$-mers ratio - see below). The results suggest that subtractive assembly can effectively detect the differential genome when the abundance ratio of the genome between two samples is about two times (or greater) the $k$-mer ratio threshold (parameter $r$ ) (on the other hand, when $r$ decreases to $<2$, significantly more reads from non-differential genomes are also extracted and subtractive assembly loses its power; Figure S1 in Additional file 1). For instance, $97.84 \%(581,047$ out of 593,858$)$ of the reads from $S$. thermophilus LMD-9 were extracted and $95.03 \%$ of the genome is covered by contigs when $r=2$ and the simulated abundance of the Streptococcus genome is four times different in abundance between the two datasets. Based on this, we conclude that the $k$ mer ratio threshold needs to be set to $r=R / 2$ to effectively assemble a genome that is about $R$ times more abundant in sample A than B, using subtractive assembly (i.e., A minus B). The simulation also suggests that the subtractive assembly approach can effectively capture genes with abundance changes of three-fold or more.

As shown above, our subtractive method can effectively recover reads originating from differential species between metagenomes. However, due to the random nature of shotgun sequencing, some regions of differential species may lack reads and are, therefore, poorly assembled, especially when the sequencing depth is not high. Here we tested subtractive assembly using simulated datasets with varying sequencing depth to demonstrate the impact of sequencing depth on the performance of subtractive assembly, using the same population structure as S1 or S4 from group 1 in simulation 1. We synthesized five pairs of datasets in which the sequencing depth ranges from 1-20× (Table 2): the sequencing depth for S1 ranges from 4-20x while it ranges from 1$5 \times$ for S4 (so in each pair of datasets, the relative abundance of S. thermophilus LMD-9 in S1 remains four times that in S4). Subtractive assembly $(r=2)$ was applied to each pair of datasets and we evaluated its performance according to the percentage of extracted reads

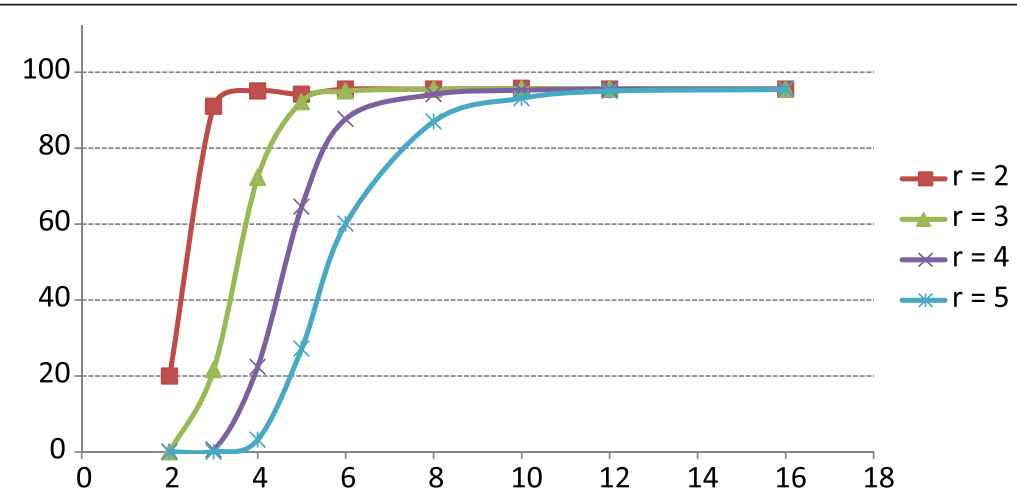

Fig. 1 Fraction of the S. thermophilus LMD-9 genome assembled using subtractive assembly with different $k$-mer ratio parameters $r$ (2-5; simulation 1). The $x$-axis shows the abundance ratio of this genome between samples and the $y$-axis shows the fraction (percentage) of the genome covered by contigs 
Table 2 Impact of sequencing depth on subtractive assembly for S. thermophilus LMD-9

\begin{tabular}{|c|c|c|c|c|}
\hline \multicolumn{2}{|c|}{ Sequencing depth } & \multirow{2}{*}{$\begin{array}{l}\text { Base } \\
\text { coverage }^{\mathrm{b}} \\
(\%)\end{array}$} & \multirow{2}{*}{$\begin{array}{l}\text { Extracted } \\
\text { reads }^{c} \\
(\%)\end{array}$} & \multirow{2}{*}{$\begin{array}{l}\text { Assembled } \\
\text { genome } \\
(\%)\end{array}$} \\
\hline$S 1^{a}$ & S4 & & & \\
\hline $4 x$ & $1 \times$ & 82.15 & 86.69 & 31.72 \\
\hline $8 x$ & $2 x$ & 86.96 & 88.93 & 67.31 \\
\hline $12 x$ & $3 x$ & 90.58 & 93.15 & 83.72 \\
\hline $16 x$ & $4 \times$ & 92.96 & 95.53 & 90.92 \\
\hline $20 x$ & $5 x$ & 94.79 & 96.91 & 93.82 \\
\hline
\end{tabular}

${ }^{a}$ The community structures of $\mathrm{S} 1$ and $\mathrm{S} 4$ are the same as in simulation 1 (group 1 in Table 1)

${ }^{\mathrm{b}}$ Expected percentage of bases with $\geq 2$ times sequencing coverage in $\mathrm{S} 1$ than in $\mathrm{S} 4$

'Percentage of reads extracted from the simulated S. thermophilus genome in $\mathrm{S} 1$

${ }^{d}$ Fraction of the genome assembled using the extracted reads by our subtractive assembly approach

and the fraction of the $S$. thermophilus genome assembled. As shown in Table 2, although the sequencing depth varies across the simulated datasets, the percentage of extracted reads was perfectly correlated with the expected ratio of differential reads $\left(R^{2}=0.9739\right)$, indicating that the performance of the subtraction step is mostly determined by the relative abundances of a genome between metagenomes. Not surprisingly, the quality of the final assembly is dependent on the sequencing depth (Fig. 2): when the sequencing coverage is low (e.g., $4 \times$ ), only a small proportion of the differential genome can be assembled; but our method recovers nearly all of the differential positions when the sequencing depth is sufficiently high (e.g., 16x).

\section{Evaluation of subtractive assembly: quality of the subtractive assembly when closely related species co-exist}

We then asked if subtractive assembly improves the assembly quality of metagenomes when closely related species exist in a sample, using another set of simulated metagenomic datasets consisting of five strains of Rhodopseudomonas palustris (Table 3). The dominant genome is $R$. palustris $\mathrm{HaA} 2$ in $\mathrm{S} 1$, while it is $R$. palustris CGA009 in S2. At the same time, the relative abundance of $R$. palustris $\mathrm{HaA} 2$ in $\mathrm{S} 2$ is substantially lower than that in S1: thus, $k$-mers representing the HaA2 genome will be identified and used for extracting reads from S1. For S1, subtractive assembly obtained longer contigs for the dominant $R$. palustris $\mathrm{HaA} 2$ genome than did direct assembly of the raw datasets, without much sacrifice of genome coverage (Fig. 3). Using contigs that are longer than 500 bp, the N50 is 21,374 in subtractive assembly, compared with 13,360 from the direct metagenomic assembly of metagenome 1; and the length of the largest contig is 113,404 bp compared with $95,495 \mathrm{bp}$. The genome coverage by contigs (total number of aligned bases in the reference divided by the genome size) is $98.3 \%$ in subtractive assembly, compared with $98.6 \%$ in direct assembly. The increased length of contigs comes with an acceptable number of misassemblies: the subtractive assembly produced three misassemblies (as reported by QUAST [29]), whereas the direct assembly produced one misassembly. The number of mismatches and indels, however, is decreased significantly in subtractive assembly of the distinctive reads: the number of mismatches is 394 with subtractive assembly and 2185 with

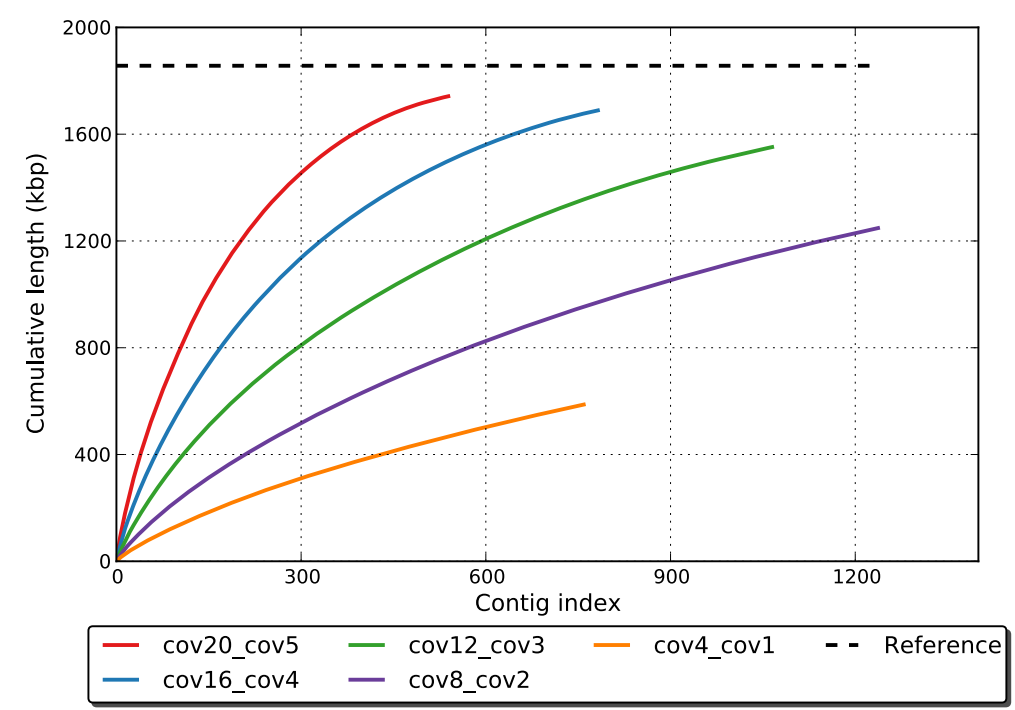

Fig. 2 Comparison of the cumulative contig length of subtractive assembly at different sequencing depths of S. thermophilus LMD-9: 20X versus $5 \times($ red), $16 \times$ versus $4 \times$ (blue), $12 \times$ versus $3 \times$ (green), $8 \times$ versus $2 \times$ (purple), $4 \times$ versus $1 \times$ (orange) 
Table 3 A pair of simulated metagenomic datasets containing five R. palustris strains (simulation 2)

\begin{tabular}{lllllll}
\hline Strain & $\begin{array}{l}\text { Genome } \\
\text { length }\end{array}$ & \multicolumn{2}{l}{$\mathrm{RA}^{\mathrm{a}}$} & & \multicolumn{2}{c}{ Sequencing depth } \\
& $\mathrm{S} 1$ & $\mathrm{~S} 2$ & & S1 & $\mathrm{S} 2$ \\
\hline BisA53 & $5,505,494$ & 3 & 3 & & $18 \times$ & $\times$ \\
BisB18 & $5,513,844$ & 3 & 3 & & $18 \times$ & $18 \times$ \\
BisB5 & $4,892,717$ & 3 & 3 & $18 \times$ & $18 \times$ \\
CGA009 & $5,459,213$ & 0.1 & 5 & & $0.6 \times$ & $30 \times$ \\
HaA2 & $5,331,656$ & 5 & 0.1 & & $30 \times$ & $0.6 \times$ \\
\hline
\end{tabular}

${ }^{a}$ Relative abundance. The two samples are S1 and S2 direct assembly; and the number of indels is 8 with subtractive assembly and 80 with direct assembly.

A possible explanation for the superior performance of subtractive assembly in this simulation is that the subtraction step helps alleviate assembly problems caused by polymorphic regions (the regions that are similar, but not identical, in multiple genomes in the same metagenomic dataset). The sharing of homologous genes among different species is one of the known complicating factors that confuse de Bruijn graph-based assemblers (including IDBA-UD [30]) in metagenomic assembly, because they form tangled branches in the assembly graph. Since subtractive assembly targets the genomes that are more abundant (or unique) in one of the metagenomes, some of the closely related genomes will

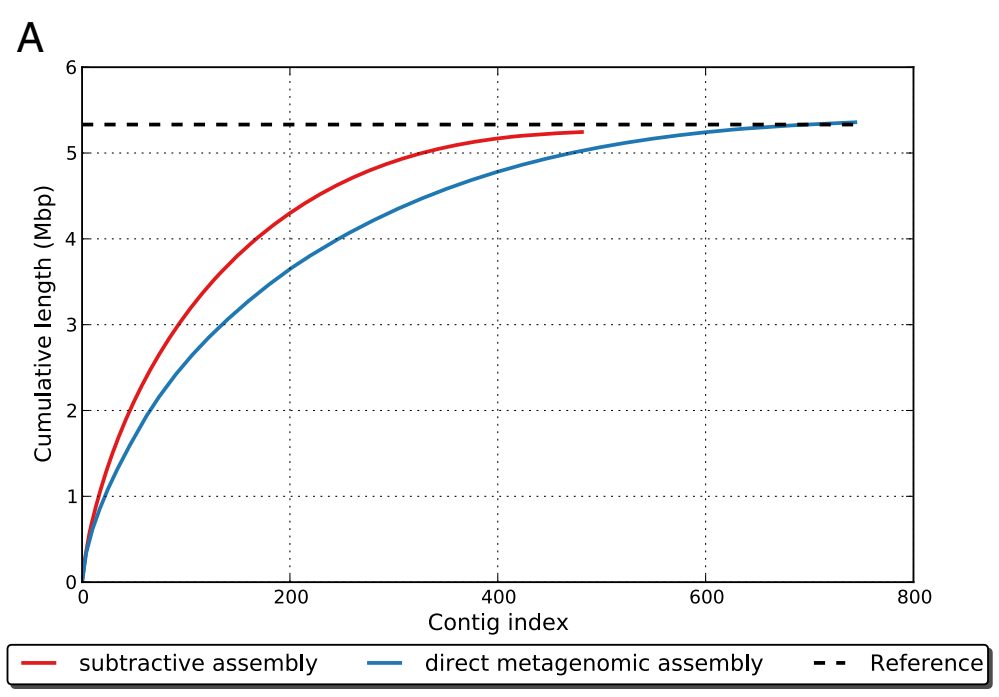

B

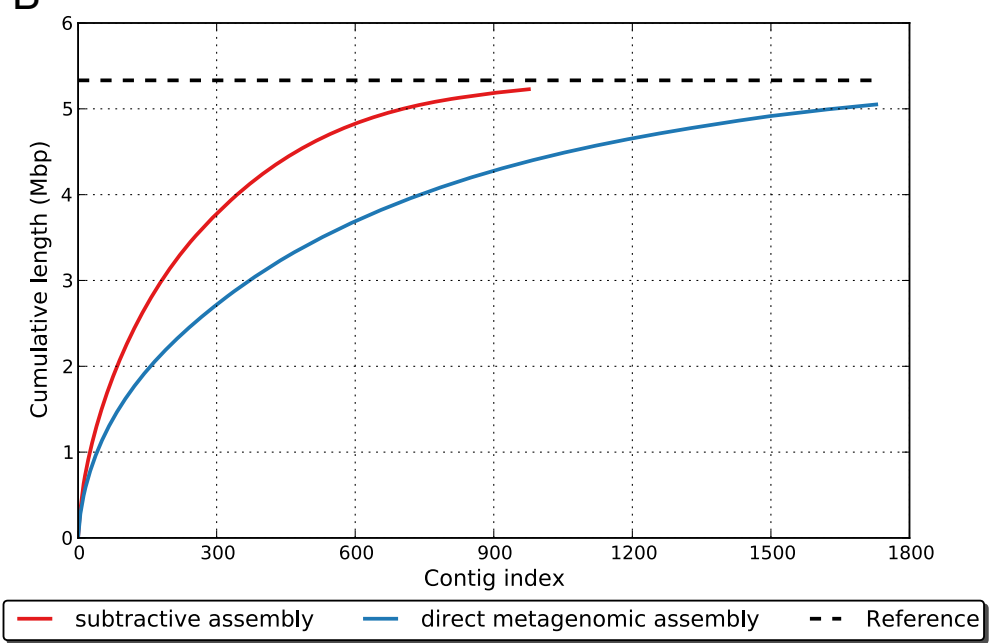

Fig. 3 Comparison of the cumulative contig length between subtractive assembly (red) and direct metagenomic assembly (blue) of $R$. palustris HaA2 (simulation 2). The results assembled by IDBA-UD and MetaVelvet are shown in (a) and (b), respectively. On the x-axis, contigs are ordered from largest to smallest. The $y$-axis gives the size of the $x$ largest contigs in the assembly 
be filtered out during the subtraction step, reducing the complexity of the assembly problem. We compared the contigs from subtractive assembly and direct assembly using NUCMER [31] and confirmed the reduced fragmentation of contigs by the subtractive assembly resulting from the subtraction step. For instance, one 43,299-bp contig from subtractive assembly was fragmented into four contigs in direct assembly (Fig. 4). Positions around breakpoints recruited a number of contigs of different degrees of similarities, indicating that these are homologous regions shared by the different genomes in the metagenomic dataset (the five strains are only moderately similar to each other at a maximal unique matches index (MUMi) distance [32] of $\sim 0.8$ ( $0 \sim 1$ scale), due to frequent genomic rearrangements).

We note that a comprehensive testing of all available assemblers is beyond the scope of this manuscript, but in addition to IDBA-UD [30] we tested MetaVelvet [33] and MEGAHIT [34]. To use MetaVelvet [33] for subtractive assembly on $\mathrm{S} 1$, we set the $k$-mer length as 51 for assembly (as suggested by the MetaVelvet manual). We saw an even greater improvement of assemblies by subtractive assembly, which is not surprising, since MetaVelvet originally generated shorter contigs for this dataset than IDBA-UD [30] (Fig. 3a, b), leaving more room for improvement. From the cumulative plot of contigs, we can see that contigs of the differential genome were longer if subtractive assembly is applied preceding metagenomic assembly. Using contigs that are longer than $500 \mathrm{bp}$, the N50 is 10,116 with subtractive assembly but only 4681 with direct metagenomic assembly, and the largest contig is increased from $76,007 \mathrm{bp}$ with direct assembly to $98,570 \mathrm{bp}$ with subtractive assembly. Even the genome coverage is improved with subtractive assembly, from $94.1 \%$ to $97.6 \%$. MEGAHIT [34] is a more recently developed assembler, which also uses the iterative assembly strategy (similar to IDBA-UD [30]). Not surprisingly, its results were comparable to those from IDBA-UD (Figure S2 in Additional file 1), but more differences were observed between these two assemblers for the real T2D gut metagenomes, as shown below.

\section{Subtractive assembly of T2D gut metagenomes}

We applied subtractive assembly to the analysis of T2D gut metagenomes, hoping to identify compositional/ functional T2D-associated features of the human microbiome, as well as test our methods with datasets of naturally occurring complexity. We used 50 T2D datasets (a total of 129 gigabases) and all 43 normal glucose tolerant (NGT) datasets (90 gigabases). We did not include three T2D datasets that were outliers based on neighborjoining clustering of the samples using a $\mathrm{d}_{2}^{\mathrm{S}}$ dissimilarity measure for $k=9$ [19]. Table 4 shows the differential reads extracted for each group of samples (T2D or NGT). A large portion of the extracted reads represented unique $k$-mers, confirming the distinction between these two groups. For comparison, we also assembled the datasets directly (without the subtractive step). We tried two different approaches: assembling the metagenomic datasets individually, or co-assembling the pooled datasets. For clarity, we call the former direct assembly, and the latter direct co-assembly.

The subtractive assembly generated fewer contigs compared with the direct co-assembly - this is not surprising because the subtractive assembly focused on the differential portion. For direct co-assembly, as the pooled dataset is huge, we could only use MEGAHIT

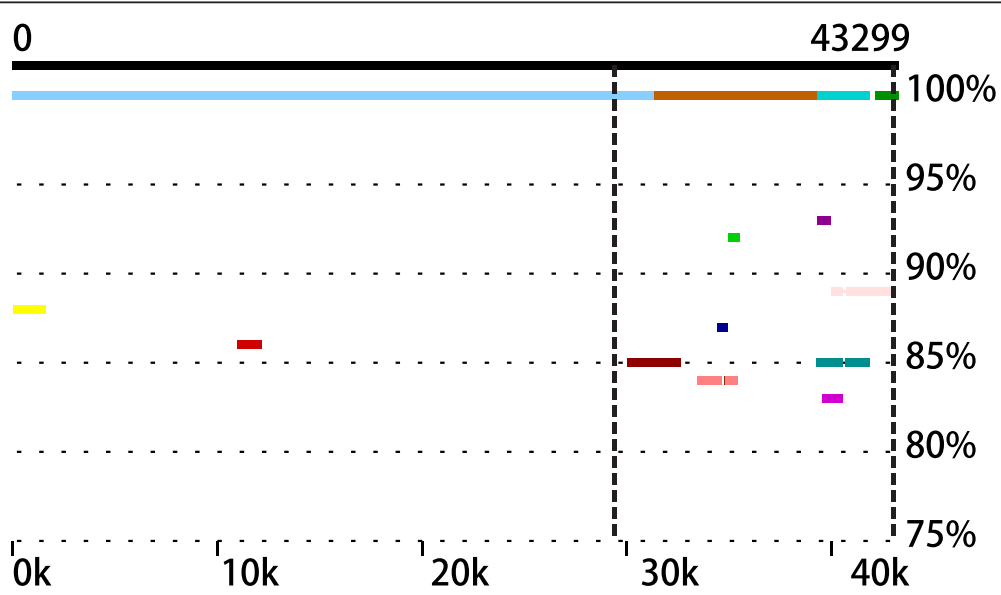

Fig. 4 An example of the reduced fragmentation of contigs given by subtractive assembly. A long contig resulting from the subtractive assembly is broken into several shorter contigs when the subtraction step is not used (i.e., from the direct assembly). A number of contigs (highlighted by different colors) from the direct assembly are aligned to the long contig with different degrees of similarities. The polymorphic region is highlighted between two vertical dotted lines 
Table 4 A summary of the read extraction for the European women gut metagenomic datasets

\begin{tabular}{lcc}
\hline k-mer ratio & NGT (gigabases) & T2D (gigabases) \\
\hline 2 & 14.48 & 12.66 \\
4 & 2.48 & 1.66 \\
6 & 0.55 & 0.42 \\
8 & 0.17 & 0.13 \\
10 & 0.04 & 0.05 \\
(unique) & 8.91 & 14.24 \\
\hline
\end{tabular}

but not IDBA-UD (which used too much memory). However, we were able to co-assemble the distinctive reads (i.e., subtractive assembly) for the combined T2D and NGT metagenomes using either assembler because of the substantial data reduction in the subtraction step. Table 5 summarizes the assembly results for the T2D samples using the direct assembly and subtractive assembly approaches (the results show similar trends for the NGT datasets). In brief, subtractive assemblies are approximately one sixth (by IDBA-UD) to one half (by MEGAHIT) the length of direct assemblies, measured as the total length of contigs; and MEGAHIT produced more contigs, but its contigs are much shorter than IDBA-UD contigs (true for both direct assembly and subtractive assembly). There is no clear assembler winner in this case, but considering that IDBA-UD gave much longer contigs (and the memory usage is not a concern for our subtractive assembly approach due to the data reduction), we focus below on the downstream application of subtractive assembly results using IDBAUD (but users can choose to use any of their favorite assemblers for subtractive assembly).

\section{Subtractive assembly reveals compositional features of T2D gut metagenomes}

To identify bacteria that are responsible for the difference between T2D and NGT gut metagenomes, we queried the contigs from subtractive assembly against the bacterial genomes (both complete and draft) deposited in National Center for Biotechnology Information (NCBI) using BLASTN [35]. MEGAN [16] was used to process the BLASTN [35] search results for taxonomic assignments of the contigs. About one half of the contigs were assigned to a reference genome in the database, and about one third of the unassigned contigs were identified by subtractive assembly but not by direct assembly. Consistent with previous studies [8], our results suggest enrichment of Lactobacillus gasseri, Lactobacillus salivarius and S. mutans in T2D datasets. However, we identified a greater variety of Lactobacillus and Streptococcus species (Fig. 5) as more abundant in the T2D group compared with the original analyses of these datasets [8]: for example, Streptococcus parasanguinis and Streptococcus salivarius are found to be enriched in the T2D datasets. We also identified genomes that are more abundant in the NGT group, including Lysinibacillus fusiformis ZC1, Lysinibacillus sphaericus C3-41, and Pseudomonas putida GB-1 (see Additional file 2: Figure S3 and Additional file 3: Figure S4 for all species that were uniquely detected in NGT and T2D, respectively). The roles of those genomes remain obscure and await further study.

Our results also show that many pathogenic bacteria (including Actinomyces, Enterococcus faecalis and Rothia mucilaginosa) are enriched in T2D datasets, which might be a consequence of the immunocompromised status of T2D patients. The association between enriched pathogens and diabetes has been consistently reported in previous studies: $42 \%$ of published cases of perianal actinomycosis were from patients also diagnosed with diabetes [36]; diabetes mellitus was identified as a unique, independent risk factor for isolation of vancomycin-resistant E. faecalis [37] and made it easier for $R$. mucilaginosa to cause infections [38]; and another large-scale metagenomics study revealed higher levels of opportunistic pathogens in participants with T2D [39].

Subtractive assembly delivers a large collection of unique or abundant genes in T2D gut metagenomes

Subtractive assembly provided us with genes that could not be (well) assembled by direct assembly of individual metagenomic samples, and we showed in simulation results that our method can improve metagenome assembly, so we further explored how this improvement would influence gene prediction and functional analysis results using the T2D datasets. Even though half of the contigs from subtractive assembly cannot be phylogenetically assigned, they could still be used for functional annotation, which may reduce the bias in the reference-based

Table 5 A summary of the subtractive assembly and direct assembly results for T2D datasets

\begin{tabular}{lllll}
\hline Metrics & Direct assembly & & \multicolumn{2}{l}{ Subtractive assembly } \\
\cline { 2 - 4 } & IDBA-UD (individual) & MEGAHIT (co-assembly) & IDBA-UD & MEGAHIT \\
\hline Total contigs ${ }^{\mathrm{b}}$ & $2,422,739$ & $2,645,944$ & 220 & $2,175,502$ \\
Total base & $3,365,389,115$ & $2,200,436,161$ & $512,470,294$ & $1,434,840,759$ \\
N50 & 2170 & 1054 & 1146 & 677 \\
\hline
\end{tabular}

${ }^{\mathrm{a}}$ The assemblies of individual samples were added for direct assembly by IDBA-UD

bonly contigs of at least $300 \mathrm{bp}$ were considered for the statistics 


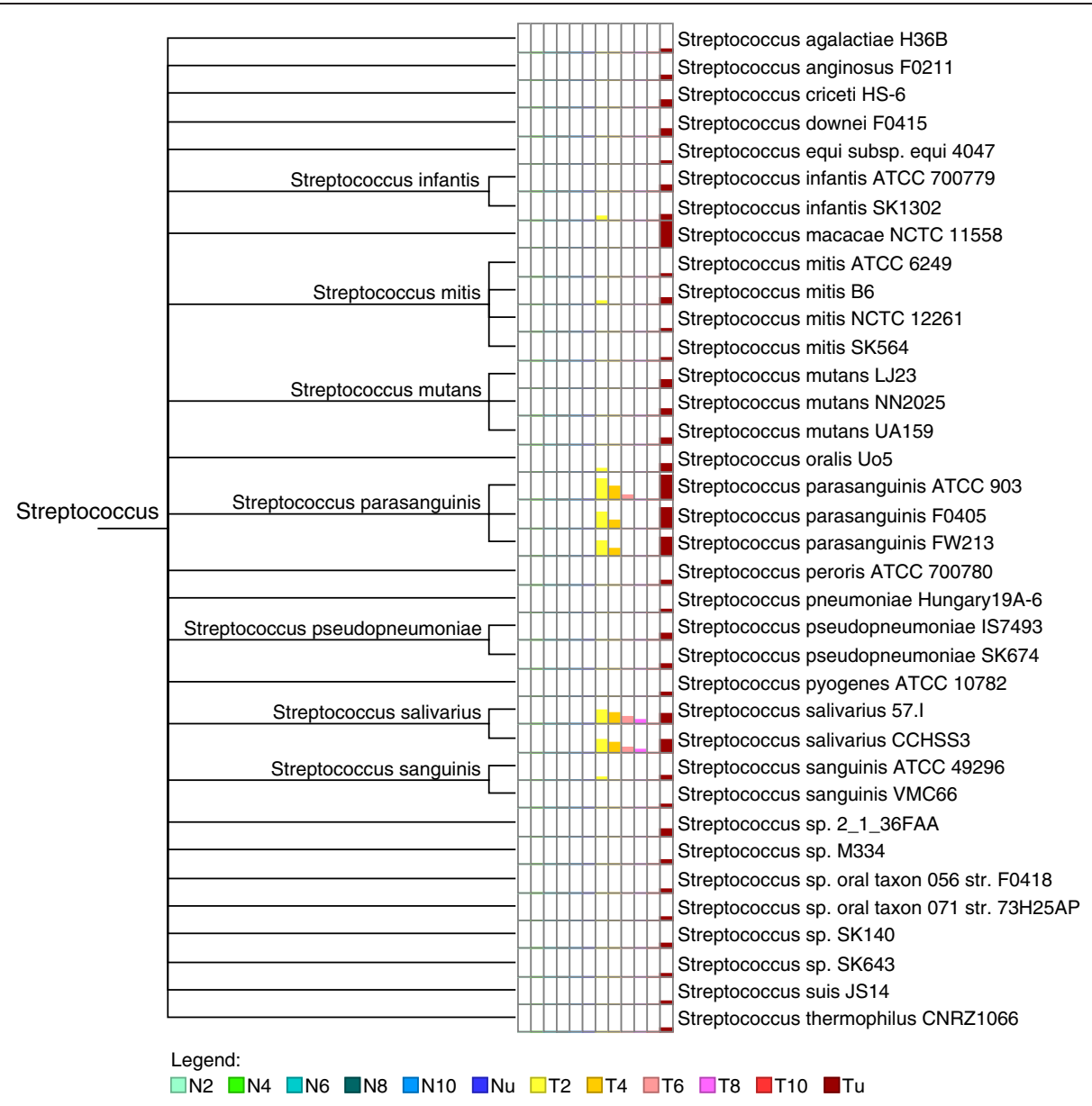

Fig. 5 Compositional differences in Streptococcus species between the T2D group and NGT group. The genomes are identified in the T2D group while absent in the NGT group, through lowest common ancestor analysis. The first six columns and last six columns represent the iterative subtractive assemblies at $k$-mer ratio 2, 4, 6, 8, and 10, or that are unique for the NGT group and T2D group, respectively. The height of the colored bars in each column is proportional to the number of contigs that hit that taxon

annotation. We compared subtractive assembly with direct assembly of individual samples (both assembled by IDBA-UD): out of 928,237 genes predicted from subtractive assembly, 141,104 genes (15\%) - among which there are 70,951 complete genes (including both a start codon and a stop codon) - cannot be found in the direct assemblies of T2D samples. Similarly, 149,321 (18 \%) - among which 72,956 genes are complete - out of 821,130 genes are not included in the direct assemblies of NGT samples. Comparison of subtractive assembly results with the coassembly results of the original datasets (both assembled by MEGAHIT) revealed improvement by subtractive assembly at comparable scales: 660,445 out of 2,978,267 genes $(22 \%)$ from subtractive assembly - among which there are 274,018 complete genes - cannot be found in the direct co-assemblies of T2D samples. Likewise, 350,997 out of 2,692,810 genes (13 \%) - among which 132,557 are complete - are not included in the direct co-assemblies of NGT samples. These results suggest that co-assembly of the datasets (thanks to the development of memory-efficient assemblers such as MEGAHIT) helped to assemble more genes compared with assembly of individual datasets; but still data reduction by subtractive assembly helped to further improve the assembly results (no matter which assembler was used).

When we compare the genes we identified with the gene sets from the original analyses of the datasets [8], we see a significant number of new genes. The original analyses [8] resulted in a collection of 5,997,383 genes from all the samples including NGT samples and T2D samples (data retrieved upon request). Using $95 \%$ sequence identity and $80 \%$ coverage of the query as cutoffs, subtractive assembly resulted in 153,755 new genes $(17 \%)$ in the T2D group and 140,542 new genes (17\%) in the NGT group.

We are particularly interested in genes that are unique or more abundant in the T2D microbiomes. We annotated these genes according to the SEED classification 
system [38]. This gene set is enriched in subsystems including peptidoglycan biosynthesis, multidrug resistance efflux pumps, and lactose and galactose uptake and utilization (Table 6). Not surprisingly, the subsystems with the most hits are involved in energy harvesting (such as lactose and galactose uptake and utilization, and fructooligosaccharides and raffinose utilization), cell defense (e.g. peptidoglycan biosynthesis and multidrug resistance efflux pumps), and transport proteins (such as Ton and Tol transport systems and ECF class transporters), indicating a microbe-contributed elevated level of glycolysis/gluconeogenesis in the T2D group, consistent with previous observations that short chain fatty acids can lead to increased glycolysis/gluconeogenesis in the liver [40,41]. We also identified sialic acid metabolism as enriched in the gut microbiome of T2D patients (Table 6); it has been reported that elevated sialic acid is strongly associated with T2D and raised serum sialic acid is a predictor of cardiovascular complications [42]. As the patients in this study are 70-year-old women, they may be in a relatively late stage of diabetes and therefore suffer from those complications.

We further narrowed down our selection of genes to those that are consistently more abundant across T2D microbiomes than in healthy controls (so can serve as dependable gene markers for T2D), considering that

Table 6 Top 20 SEED subsystems for genes identified uniquely by subtractive assembly in the T2D cohort

\begin{tabular}{lll}
\hline Rank & SEED subsystem & Number of genes \\
\hline 1 & Peptidoglycan biosynthesis & 635 \\
2 & Ton and Tol transport systems & 451 \\
3 & Multidrug resistance efflux pumps & 427 \\
4 & DNA replication & 424 \\
5 & DNA repair, bacterial & 364 \\
6 & Cell division subsystem & 345 \\
7 & Lactose and galactose uptake and utilization & 322 \\
8 & Restriction-modification system & 322 \\
9 & Fructooligosaccharides and raffinose utilization & 292 \\
10 & Glycerolipid and glycerophospholipid & 276 \\
& metabolism & \\
11 & Maltose and maltodextrin utilization & 270 \\
12 & Sialic acid metabolism & 257 \\
13 & Methionine degradation & 253 \\
14 & Ribosome LSU bacterial & 252 \\
15 & Glycolysis and gluconeogenesis & 244 \\
16 & De novo pyrimidine synthesis & 238 \\
17 & High affinity phosphate transporter & 236 \\
18 & ECF class transporter & 234 \\
19 & Purine conversion & Threonine and homoserine biosynthesis \\
\hline
\end{tabular}

subtractive assembly can improve the assembly of those genes. To identify those consistently differential genes, we quantified the abundance of the genes using readmapping (by BWA [43]), normalized by the total number of reads (per billion reads) in each sample, to identify the genes that are significantly enriched in the T2D group compared with the NGT group. Among the 141,104 differential genes that cannot be found in direct assemblies of T2D samples, 18,614 (13\%) were significantly enriched in all T2D samples, with q-value $<0.01$ (Wilcoxon rank-sum test corrected by false discovery rate (FDR)). Although we observed similar rankings for the top subsystems, we saw increases in subsystems related to energy harvesting (e.g., the rank for the 'fructooligosacchrides and raffinose utilization' subsystem was increased from 7 to 2) using this more stringent collection of T2D differential genes that passed the multiple testing (Table 7). We list significantly T2D-enriched genes together with their annotations on our website (http://omics.informatics.indiana.edu/mg/SA/).

\section{Example T2D signature subsystems and genes}

Here we present a few involved subsystems and genes in detail. The first three subsystems involve utilization of fructooligosaccharides (FOS), maltose, lactose and galactose, and they are enriched in T2D women (ranked as 2, 11, and 13 in Table 7). For 11 out of 16 functional roles involved in the 'Fructooligosaccarides and riffinose utilization' subsystem, genes with differential abundances were identified (Table S1 in Additional file 1); detailed analysis of FIGfams in these three subsystems revealed

Table 7 Top 13 SEED subsystems for genes identified uniquely by subtractive assembly, and which passed a Wilcoxon rank-sum test with FDR correction, in the T2D cohort

\begin{tabular}{lll}
\hline Rank & SEED subsystem & $\begin{array}{l}\text { Number of } \\
\text { genes }\end{array}$ \\
\hline 1 & Peptidoglycan biosynthesis & 138 \\
2 & Fructooligosaccharides and raffinose utilization & 103 \\
3 & Multidrug resistance efflux pumps & 100 \\
4 & Cell division & 89 \\
5 & Sialic acid metabolism & 80 \\
6 & Gene cluster associated with Met-tRNA & 77 \\
7 & formyltransferase & \\
& Glycerolipid and glycerophospholipid & 73 \\
8 & metabolism & 73 \\
9 & Cha repair, bacterial & 73 \\
& biosynthesis & \\
10 & Murein hydrolases & 72 \\
11 & Maltose and maltodextrin utilization & 66 \\
12 & Beta-glucoside metabolism & 62 \\
13 & Lactose and galactose uptake and utilization & 62 \\
\hline
\end{tabular}


an enrichment of several glycosidases with various substrate specificities (EC 3.2.1.-). For the utilization of FOS, there are at least three glycosidases with elevated levels in T2D: beta-glucosidase (EC 3.2.1.21), alphagalactosidase (EC 3.2.1.22) and alpha-mannosidase (EC 3.2.1.24); for the utilization of lactose and galactose, beta-galactosidase (EC 3.2.1.23) is significantly increased in the T2D cohort (Fig. 6); similarly, alpha-glucosidase (EC 3.2.1.20) is increased, for enhanced utilization of maltose. We note that alpha-glucosidase inhibitors are well-established in the treatment of T2D, and work by reducing the absorption of carbohydrates from the small intestine [44]. Our work revealed other enriched glycosidases in T2D, which may provide alternative targets for the development of antidiabetic drugs.

The next two genes, truB (T2D_unique_8729_300_ 1012_+) and ribF (T2D_unique_8729_1193_2032_+), were found in the same contig assembled by subtractive assembly. The truB gene encodes the pseudouridylate synthase TruB (PF01509; 239 amino acids), and the ribF gene encodes a prokaryotic riboflavin biosynthesis protein (PF06574; 278 amino acids); the gene product of ribF has both flavokinase and adenine dinucleotide synthetase (FAD synthetase) activities (Fig. 7a). Flavokinases (EC 2.7.1.26) catalyze the conversion of riboflavin to FMN, while FAD synthetase (EC 2.7.7.2) adenylates FMN to FAD, together converting riboflavin to the catalytically active cofactors FMN and FAD [45]. By blasting

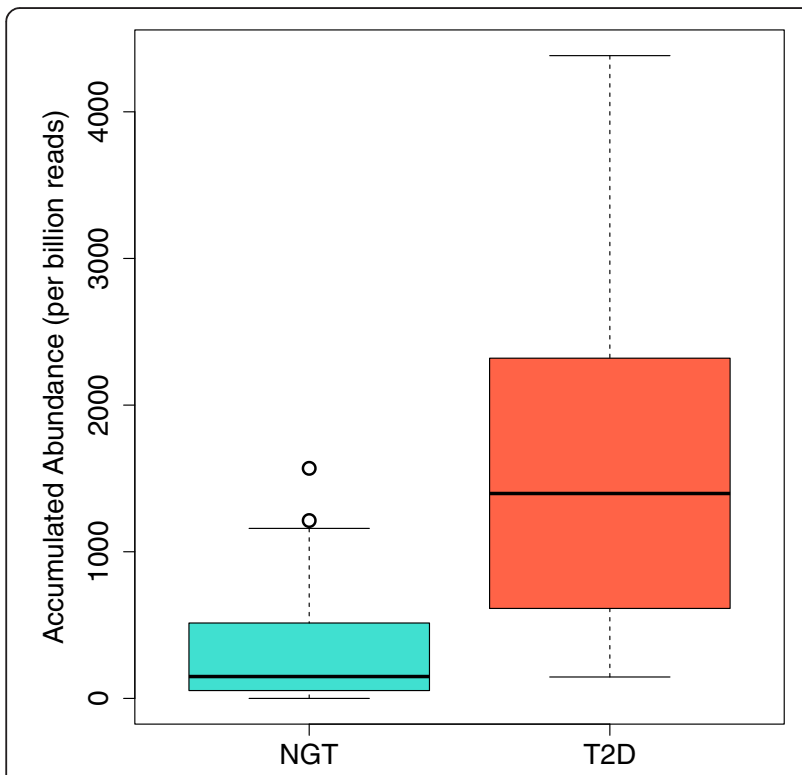

Fig. 6 Abundance difference of the genes encoding betagalactosidase between T2D and normal microbiomes (NGT). The abundance was measured as the number of reads that can be mapped to significantly T2D-enriched beta-galactosidase-encoding genes per billion reads. Note that we excluded 3 of 50 T2D samples with overly abundant beta-galactosidase genes (abundance $>6000$ ) from the plot for clarity the genes against the NR database [46], we identified the source genome to be Blautia sp. CAG:257 with 99 $\%$ identity and $98 \%$ coverage of the query sequence. Karlsson et al. [8] also reported an abnormal level of riboflavin metabolism in the gut microbiome of T2D patients; however, they claimed that riboflavin metabolism was enriched in NGT women. We notice that their results may actually indicate the opposite (and so be consistent with our conclusion): they identified three KEGG (Kyoto Encyclopedia of Genes and Genomes) [47] protein families (KEGG Orthology groups) involved in riboflavin metabolism increased in NGT, while six other protein families were more abundant in T2D (shown in their supplementary table 12) [8]. The contig containing these genes was assembled from reads 'unique' to the T2D samples; read mapping confirmed that only a very few reads (59) from the NGT samples can be mapped to this 3450-bp contig (in contrast, 521 reads from T2D microbiomes can be mapped to this contig; Fig. 7b). This increase in FMN and FAD synthetase is consistent with the increased energy harvesting suggested above: FAD helps extract chemical energy by taking electrons from glucose during oxidative respiration.

The last gene (T2D_unique_70674_105_963_+) encodes a 285 amino acid protein with only one domain: MATE (PF01554; Multi antimicrobial extrusion protein). The protein belongs to one of the ten protein families (FIGfams) associated with the Multidrug resistance efflux pumps subsystem. This FIGfam (FIG 00000402) has the most hits for differential genes (342/427) among the ten FIGfams; members of this protein family extrude cationic drugs through an $\mathrm{Na}^{+}$-coupled antiport mechanism [48]. Taxonomic assignments of these proteins indicate a Firmicutes origin, especially Clostridium, Lachnospiraceae and Erysipelotrichaceae. It is known that mammalian MATE transporters mediate multidrug resistance by exporting diverse xenobiotic cations in the liver and kidney (MATE1 protein, for example, reduces the plasma concentrations of metformin, a widely prescribed oral glucose-lowering drug for the treatment of T2D, modulating its therapeutic efficacy $[49,50]$ ), while bacterial MATE transporters act primarily as xenobiotic efflux pumps and have been reported to confer tigecycline resistance $[48,51,52]$. The elevated level of bacterial MATE pumps in the gut of T2D patients suggests a potential link between the disease and the gut microbiome through the elevated levels of medications, including antibiotics, taken by T2D patients [53, 54].

\section{Conclusions}

Using both simulated and real metagenomes, we have shown that subtractive assembly improves the assembly of the differential genome between two metagenomes and facilitates downstream analysis. If the short reads from 
A
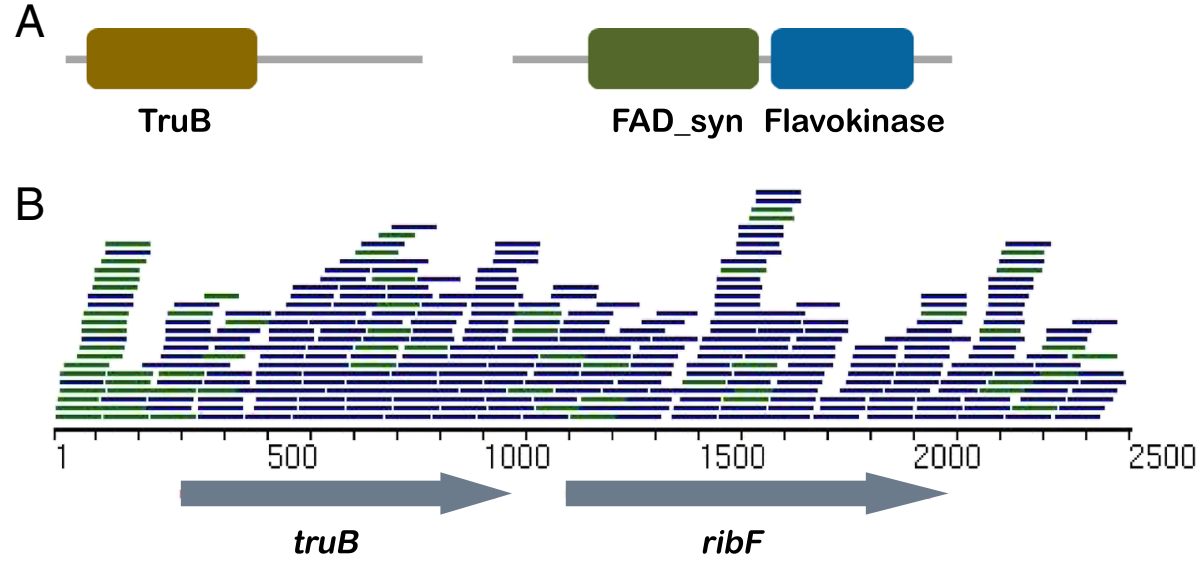

Fig. 7 The truB-ribF operon identified by subtractive assembly as associated with T2D. a There are three domains in the operon: TruB encoded by truB; and flavokinase and FAD synthetase encoded by ribF. The flavokinase and FAD synthetase constitute the bifunctional prokaryotic riboflavin biosynthesis protein. $\mathbf{b}$ The genes truB and ribF in this operon are confirmed by read mapping. Reads mapped to the proper pair are colored in blue and mapped singletons are colored in green

many genomes are directly assembled and annotated, it takes a tremendous amount of computational resources, as well as degrading the quality of the assembly. As a result, traditional comparative metagenomic approaches assemble each of the metagenomic samples independently, and then compare groups of samples by the common features shared among samples in each group. Instead, our method focuses on the compositional difference of the metagenome sets to be compared and therefore is well suited for large-scale comparative studies. Our method is able to consider a large number of samples simultaneously, which can also improve the assembly of differential genes, providing a complementary solution to existing comparative metagenomic approaches. We note that our subtractive assembly approach can effectively assemble genes with only an abundance difference of threefold or greater. However, genes with subtler abundance differences can still be discovered through the traditional comparative analyses of metagenomic datasets (using direct assembly approaches).

We developed our iterative subtractive assembly strategy to deal with situations where the compositional differences between metagenomes are unknown - which is typical. One advantage of this strategy is that it samples a spectrum of differences, aiding the assembly of genomes that are differential at various levels. However, if a user is interested in a certain degree of difference, a fixed $k$-mer ratio cutoff can be used in the subtractive assembly.

Our method currently compares two categories of metagenomes. It proves to be useful when we compare microbial communities between two treatment groups (such as healthy- versus T2D-hosted metagenomes). One future direction is to extend the method to allow comparison of multiple classes/treatments of metagenomes (e.g., sampled from multiple time points from the same environment; a control group and alternative treatments). A simple strategy is to apply subtractive assembly to all pairs of sample sets and then combine the results. We will also explore other approaches - for example, by correlating $k$-mers based on their frequency spectrum across samples for subtractive assembly - to make the best use of the multiple metagenomic datasets. Our approach to selecting consistently abundant genes related to T2D from differential genes assembled by subtractive assembly helps to narrow the gene list to the most promising ones (which are consistently differential between the two conditions according to the Wilcoxon rank-sum test with FDR correction).

Our analysis of T2D-hosted metagenomes indicates that subtractive assembly has a greater ability to detect differences than did previous analysis of the same data sets. But in general, we confirm that T2D-associated metagenomes have an increased ability to harvest energy from diverse carbohydrates, as other studies have shown. The enrichment of various glycosidases in T2D microbiomes suggests alternative targets for the development of antidiabetic drugs (alpha-glucosidase inhibitors are well-established in the treatment of T2D). The prevalence of Blautia sp. metabolism and Firmicutes-associated MATE xenobiotic efflux pumps seem to be exciting leads deserving of further study. We believe that our subtractive assembly approach can be applied to other datasets (e.g., the more recent liver cirrhosis datasets [55]) to reveal the association between microbial communities and other human diseases.

\section{Materials and methods}

Counting $k$-mers with the aid of a bloom filter

The bloom filter is a probabilistic data structure for determining whether an element belongs to a sparse set 
[23, 24, 56], using a number of hash functions to map the elements to the fixed bit space of the filter. Thus, false positives can occur when the bits for an element are shared by other elements. In other words, the bloom filter is a trade-off between memory usage and allowable false positives: suppose $n k$-mers are stored in a bitmap of size $m$ using $d$ hash functions, then the optimal value of $d$ that minimizes the false positive rate is $(m / n) \ln (2)$ $[22,57]$. As a fixed number of bits are used for each element, the complexity of inserting or querying an element is constantly $\mathrm{O}(\mathrm{d})$.

Bloom filters are memory efficient; however, the actual memory usage depends on the hash tables used for recording the number of occurrences of each $k$-mer. We modified the implementation of BFCounter (version 0.2) [22], following their principle of ruling out singletons of all $k$-mers encountered. A bloom filter $B$ and a simple hash table $T$ are adopted to store and count $k$-mers. The bloom filter $B$ is used to store all existing $k$-mers, of which only $k$-mers observed twice or more are inserted into the hash table $T$. With the information stored in hash table $T$, we are able to calculate the distinctive sequence signatures for each metagenome. To detect the compositional differences of compared metagenomes, a $k$-mer ratio parameter $r$ is employed to filter for $k$-mers that are more abundant or unique in one of the metagenomes. For example, if we set $r=10$, we will only keep $k$-mers that occur at least ten times more frequently in metagenome A compared with metagenome B as differential $k$-mers representing metagenome $\mathrm{A}$; the genomic differences between the two metagenomes are likely to be built using those signatures. We note that $k$-mer counts are normalized by the total bases in the corresponding metagenomic dataset, so that the $k$-mer ratio is not biased toward the larger metagenomic dataset.

\section{Read extraction based on sequence signatures and subtractive assembly}

Reads made up of differential $k$-mers are from genomes that are most associated with the environmental conditions of interest (assuming a lack of confounding differences). Maillet et al. [18] considered two sequences similar if and only if they share at least a number of non-overlapping $k$-mers. Different from their approach, here we define reads containing at least a certain percentage (default $50 \%$ ) of differential $k$-mers as the distinctive reads. The reads satisfying this requirement are extracted and employed for metagenomic assembly.

IDBA-UD (version 1.0.9) [30] was adopted as the metagenomic assembler, following read extraction in subtractive assembly. It has been demonstrated that IDBA-UD achieves longer contigs with higher accuracy by taking into consideration the uneven sequencing depth of metagenomic sequencing technologies [29, 30]. We adopted the default options for IDBA-UD's parameter settings: a minimum $k$-mer size of 20 and maximum $k$-mer size of 100 , with 20 increments in each iteration. For comparison purposes, we also used IDBA-UD (using the same set of parameters) to assemble individual metagenomes without applying the subtraction step (referred to as the direct assembly approach). In addition, we tested MetaVelvet (version 1.1.01) [33] and MEGAHIT (version 0.2.1) [34]. In principle, however, any metagenomic assembler can be used for subtractive assembly.

\section{Iterative subtractive assembly}

When subtractive assembly is applied to real metagenomic samples, we may choose a small $k$-mer ratio cutoff (e.g., 2), due to the unknown degree of compositional difference between the groups of samples being compared. Alternatively, we can iteratively extract reads using a series of $k$-mer ratio cutoffs. For the gut metagenomic datasets used in our study, the maximum ratio was set to 10 and the minimum 2, with a step value of 2 . Besides this, we separately extracted reads characterized by unique $k$-mers $(k$-mers that occur in only one of the groups of samples): unique $k$-mers were first identified in each group and the corresponding distinctive reads were extracted; then non-unique $k$-mers that were more frequent in one group than the other were identified and the distinctive reads were extracted, starting from a $k$ mer ratio of 10 , then 8 , and so on. The stratification by iterative assembly provides more information on the compositional difference between two metagenomes, without any prior knowledge.

\section{Annotation of contigs from subtractive assembly}

Contigs that are at least 300 nucleotides long were phylogenetically annotated by query against the bacterial genomes (both complete and draft genomes) deposited in the NCBI through BLAST searches [35]. BLAST results were then used for the assignment of lowest common ancestor by MEGAN (version 4) [16], with a minimum bit score (Min Score) of 80 and minimum contig support (Min Support) of 5.

Protein coding genes were predicted from the contigs using FragGeneScan [58]. We are interested in the protein coding genes covered only by subtractive assembly, and consider that a gene belongs to this category if there is no equivalent gene that covers at least $20 \%$ of the gene with $90 \%$ or higher sequence identity (based on RAPSearch2 [59]) in the direct assemblies of any individual metagenome. These genes were assigned to functional categories, including SEED subsystems [60]. We used myRAST (version 36; downloaded from http://blog.the seed.org/downloads/myRAST-Intel.dmg) for the SEED subsystem annotation. 
To further validate the differential genes, we mapped the original short reads of each sample onto the genes that are enriched in the T2D cohort and normalized the coverage by the total number of reads in each sample. Based on the coverage of each differential gene in each sample, the significance of each candidate differential gene was tested by computing a one-sided $p$ value using the $\mathrm{R}$ 'wilcox.test' function and correcting for multiple testing using false discovery rate ( $q$-value) computed by the tail area-based method of the $\mathrm{R}$ 'fdrtool' package [61]. The fdrtool has been used for similar purposes in metagenomics projects [62-64].

\section{Simulated metagenomes}

We carried out two simulations to test if our subtractive assembly approach can efficiently detect compositional differences between metagenomes (and the minimum abundance ratio for the difference to be detected), and improve assembly quality (especially when closely related species co-exist in a community).

In simulation 1, we simulated three groups of metagenomic datasets using five bacterial genomes from the FAMeS dataset [65]: Ferroplasma acidarmanus fer1, Lactobacillus gasseri ATCC 33323, Pediococcus pentosaceus ATCC 25745, Prochlorococcus marinus NATL2A, and S. thermophilus LMD-9. MetaSim (version 0.9.1) [66] was used to simulate reads from the genomes. In each group, the first sample (S1) was compared with each of the remaining samples in the same group for subtractive assembly. The relative abundances of the five genomes in each sample are shown in Table 1. In these samples, we only changed the abundances of the $S$. thermophilus genome and another genome, to keep the ratio of relative abundance for the $S$. thermophilus genome in the range of 2-16. This enables us to evaluate whether our method can effectively detect the compositional difference between metagenomes by focusing on a single genome ( $S$. thermophilus). We applied the iterative subtractive assembly strategy to analyze this set of simulated datasets $(k$-mer ratio parameter $r$ was set to be $2,3,4$, or 5). After the subtractive assembly, we calculated the fraction of the S. thermophilus genome covered by contigs using QUAST [29] and MUMer [31]. In all the samples, the sequencing depth of the Streptococcus genome was designed to be between $30 \times$ and $40 \times$.

In simulation 2, we simulated a pair of metagenomic samples (S1 and S2) using five different $R$. palustris strains (Table 2). The $R$. palustris HaA2 genome is dominant in sample 1 (S1) and is the focus of this simulation. We set the $k$-mer ratio parameter to $r=2$ for the subtractive assembly (S1 minus S2). Sample 1 was also used for direct metagenomic assembly using IDBA-UD [30]. Assemblies from both subtractive assembly and direct assembly were compared with the reference genomes using QUAST [29]. We used various metrics for assembly evaluation, including the cumulative length of contigs, N50, and size of the largest contig.

\section{Real metagenomes}

We chose the large collection of gut metagenomic datasets derived from two groups of 70-year-old European women, one group of 53 with $\mathrm{T} 2 \mathrm{D}$ and the other a matched group of healthy controls (NGT group; 43 participants) [8]. This collection of metagenomes is ideal for testing our subtractive assembly approach: only two groups were involved (T2D versus healthy) and each group contains many large metagenomic datasets. We pooled the T2D samples and NGT samples separately for subtractive assembly.

\section{Availability}

Our tools for subtractive assembly are available for download at sourceforge (https://sourceforge.net/projects/ subtractive-assembly/). We also make available the subtractive assembly results of the T2D metagenomes, including the set of genes that are uniquely or more abundantly found in T2D genomes, along with their annotations at http://omics.informatics.indiana.edu/mg/SA/.

\section{Additional files}

\begin{abstract}
Additional file 1: Table S1. Functional roles involved in the 'Fructooligosaccharides (FOS) and Raffinose Utilization' subsystem. Figure S1. Percentage of extracted reads from non-differential genomes by subtractive assembly on S1 vs. S2 in Group 2 of Simulation 1 (see Table 1 for more information). The $x$-axis shows the values of $k$-mer ratio parameter $r$ (2 to 5 ) and $y$-axis shows the fraction (\%) of reads from non-differential genomes in the extracted reads. Figure $\mathbf{S} \mathbf{2}$ Comparison of the cumulative contig length between subtractive assembly (red) and direct assembly (blue) of R. palustris HaA2 (Simulation 2) by using MEGAHIT as the assembler. On the x-axis, contigs are ordered from largest to smallest. The $y$-axis gives the size of the $x$ largest contigs in the assembly. (PDF $124 \mathrm{~kb}$ )
\end{abstract}

Additional file 2: Figure $\mathbf{5 3}$. Species uniquely detected in the NGT group. (PDF $116 \mathrm{~kb}$ )

Additional file 3: Figure S4. Species uniquely detected in the T2D group. (PDF $141 \mathrm{~kb}$ )

\section{Abbreviations}

bp: base pair; FDR: false discovery rate; FOS: fructooligosaccharide; NCBI: National Center for Biotechnology Information; NGT: normal glucose tolerance; T2D: type 2 diabetes.

\section{Competing interests}

The authors declare that they have no competing interests.

\section{Authors' contributions}

MW participated in the design of the study, implemented the software, carried out the analysis and drafted the manuscript. TD participated in the analysis and helped to draft the manuscript. YY conceived the study, and participated in its design and coordination, participated in the analysis, and helped to draft the manuscript. All authors have read and approved the final manuscript. 


\section{Acknowledgements}

This work was supported by National Science Foundation (grant number DBI-0845685) and National Institute of Health (grant number 1R01Al108888-01A1).

\section{Author details}

${ }^{1}$ School of Informatics and Computing, Indiana University, Bloomington, IN 47405, USA. ²Department of Biology, Indiana University, Bloomington, IN 47405, USA. ${ }^{3}$ National Center for Genome Analysis Support, Indiana University, Bloomington, IN 47401, USA.

\section{Received: 22 August 2014 Accepted: 9 October 2015}

\section{Published online: 02 November 2015}

\section{References}

1. Wooley JC, Ye Y. Metagenomics: facts and artifacts, and computational

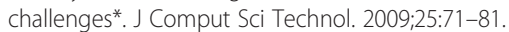

2. Galperin MY. Metagenomics: from acid mine to shining sea. Environ Microbiol. 2004;6:543-5.

3. Human Microbiome Project C. Structure, function and diversity of the healthy human microbiome. Nature. 2012;486:207-14.

4. Gnerre S, Maccallum I, Przybylski D, Ribeiro FJ, Burton JN, Walker BJ, et al. High-quality draft assemblies of mammalian genomes from massively parallel sequence data. Proc Natl Acad Sci U S A. 2011;108:1513-8.

5. Treangen TJ, Koren S, Sommer DD, Liu B, Astrovskaya I, Ondov B, et al. MetAMOS: a modular and open source metagenomic assembly and analysis pipeline. Genome Biol. 2013;14:R2.

6. Mende DR, Waller AS, Sunagawa S, Jarvelin Al, Chan MM, Arumugam M, et al. Assessment of metagenomic assembly using simulated next generation sequencing data. PLoS One. 2012;7, e31386.

7. Nagarajan N, Pop M. Sequence assembly demystified. Nat Rev Genet. 2013;14:157-67.

8. Karlsson FH, Tremaroli V, Nookaew I, Bergstrom G, Behre CJ, Fagerberg B, et al. Gut metagenome in European women with normal, impaired and diabetic glucose control. Nature. 2013;498:99-103.

9. Arndt D, Xia J, Liu Y, Zhou Y, Guo AC, Cruz JA, et al. METAGENassist: a comprehensive web server for comparative metagenomics. Nucleic Acids Res. 2012;40:W88-95.

10. Tringe SG, von Mering C, Kobayashi A, Salamov AA, Chen K, Chang HW, et al. Comparative metagenomics of microbial communities. Science. 2005;308:554-7.

11. Modi SR, Lee HH, Spina CS, Collins JJ. Antibiotic treatment expands the resistance reservoir and ecological network of the phage metagenome. Nature. 2013;499:219-22.

12. Sangwan N, Lata P, Dwivedi V, Singh A, Niharika N, Kaur J, et al. Comparative metagenomic analysis of soil microbial communities across three hexachlorocyclohexane contamination levels. PLoS One. 2012;7, e46219.

13. Steffen MM, Li Z, Effler TC, Hauser L, Boyer GL, Wilhelm SW. Comparative metagenomics of toxic freshwater cyanobacteria bloom communities on two continents. PLoS One. 2012;7, e44002.

14. Xie W, Wang F, Guo L, Chen Z, Sievert SM, Meng J, et al. Comparative metagenomics of microbial communities inhabiting deep-sea hydrothermal vent chimneys with contrasting chemistries. ISME J. 2011;5:414-26.

15. Wang Y, Leung HC, Yiu SM, Chin FY. MetaCluster 4.0: a novel binning algorithm for NGS reads and huge number of species. J Comput Biol. 2012;19:241-9.

16. Huson DH, Mitra S, Ruscheweyh HJ, Weber N, Schuster SC. Integrative analysis of environmental sequences using MEGAN4. Genome Res. 2011;21:1552-60

17. Markowitz VM, Chen IM, Chu K, Szeto E, Palaniappan K, Grechkin Y, et al. IMG/M: the integrated metagenome data management and comparative analysis system. Nucleic Acids Res. 2012;40:D123-9.

18. Maillet N, Lemaitre C, Chikhi R, Lavenier D, Peterlongo P. Compareads: comparing huge metagenomic experiments. BMC Bioinformatics. 2012;13 Suppl 19:S10.

19. Jiang B, Song K, Ren J, Deng M, Sun F, Zhang X. Comparison of metagenomic samples using sequence signatures. BMC Genomics. 2012;13:730

20. Karlin S, Mrazek J, Campbell AM. Compositional biases of bacterial genomes and evolutionary implications. J Bacteriol. 1997;179:3899-913.
21. Marcais G, Kingsford C. A fast, lock-free approach for efficient parallel counting of occurrences of k-mers. Bioinformatics. 2011;27:764-70.

22. Melsted P, Pritchard JK. Efficient counting of $k$-mers in DNA sequences using a bloom filter. BMC Bioinformatics. 2011;12:333.

23. Pell J, Hintze A, Canino-Koning R, Howe A, Tiedje JM, Brown CT. Scaling metagenome sequence assembly with probabilistic de Bruijn graphs. Proc Natl Acad Sci U S A. 2012;109:13272-7.

24. Stranneheim $\mathrm{H}$, Kaller M, Allander T, Andersson B, Arvestad L, Lundeberg J. Classification of DNA sequences using Bloom filters. Bioinformatics. 2010;26:1595-600.

25. Hintao J, Teanpaisan R, Chongsuvivatwong V, Ratarasan C, Dahlen G. The microbiological profiles of saliva, supragingival and subgingival plaque and dental caries in adults with and without type 2 diabetes mellitus. Oral Microbiol Immunol. 2007;22:175-81.

26. Angelakis $E$, Raoult $D$. The increase of Lactobacillus species in the gut flora of newborn broiler chicks and ducks is associated with weight gain. PLOS One. 2010;5, e10463.

27. Armougom F, Henry M, Vialettes B, Raccah D, Raoult D. Monitoring bacterial community of human gut microbiota reveals an increase in Lactobacillus in obese patients and Methanogens in anorexic patients. PLoS One. 2009;4, e7125.

28. Musso G, Gambino R, Cassader M. Interactions between gut microbiota and host metabolism predisposing to obesity and diabetes. Annu Rev Med. 2011;62:361-80.

29. Gurevich A, Saveliev V, Vyahhi N, Tesler G. QUAST: quality assessment tool for genome assemblies. Bioinformatics. 2013;29:1072-5.

30. Peng Y, Leung HC, Yiu SM, Chin FY. IDBA-UD: a de novo assembler for single-cell and metagenomic sequencing data with highly uneven depth. Bioinformatics. 2012;28:1420-8.

31. Delcher AL, Phillippy A, Carlton J, Salzberg SL. Fast algorithms for large-scale genome alignment and comparison. Nucleic Acids Res. 2002;30:2478-83.

32. Deloger M, El Karoui M, Petit MA. A genomic distance based on MUM indicates discontinuity between most bacterial species and genera. J Bacteriol. 2009;191:91-9.

33. Namiki T, Hachiya T, Tanaka H, Sakakibara Y. MetaVelvet: an extension of Velvet assembler to de novo metagenome assembly from short sequence reads. Nucleic Acids Res. 2012;40, e155.

34. Li D, Liu CM, Luo R, Sadakane K, Lam TW. MEGAHIT: an ultra-fast single-node solution for large and complex metagenomics assembly via succinct de Bruijn graph. Bioinformatics. 2015;31:1674-6.

35. Altschul SF, Madden TL, Schaffer AA, Zhang J, Zhang Z, Miller W, et al. Gapped BLAST and PSI-BLAST: a new generation of protein database search programs. Nucleic Acids Res. 1997;25:3389-402.

36. Coremans G, Margaritis V, Van Poppel HP, Christiaens MR, Gruwez J, Geboes $\mathrm{K}$, et al. Actinomycosis, a rare and unsuspected cause of anal fistulous abscess: report of three cases and review of the literature. Dis Colon Rectum. 2005;48:575-81.

37. Hayakawa K, Marchaim D, Palla M, Gudur UM, Pulluru H, Bathina P, et al. Epidemiology of vancomycin-resistant Enterococcus faecalis: a case-case-control study. Antimicrob Agents Chemother. 2013;57:49-55.

38. Michels F, Colaert J, Gheysen F, Scheerlinck T. Late prosthetic joint infection due to Rothia mucilaginosa. Acta Orthop Belg. 2007;73:263-7.

39. Qin J, Li Y, Cai Z, Li S, Zhu J, Zhang F, et al. A metagenome-wide association study of gut microbiota in type 2 diabetes. Nature. 2012;490:55-60.

40. Veech RL, Gitomer WL, King MT, Balaban RS, Costa JL, Eanes ED. The effect of short chain fatty acid administration on hepatic glucose, phosphate, magnesium and calcium metabolism. Adv Exp Med Biol. 1986;194:617-46.

41. den Besten G, Lange K, Havinga R, van Dijk TH, Gerding A, van Eunen K, et al. Gut-derived short-chain fatty acids are vividly assimilated into host carbohydrates and lipids. Am J Physiol Gastrointest Liver Physiol. 2013;305:G900-10.

42. Rahman IU, Malik SA, Bashir M, Khan RU, Idrees M. Serum sialic acid changes in type 2 diabetic patients on metformin or rosiglitazone treatment. J Clin Pharm Ther. 2010;35:685-90.

43. Li H, Durbin R. Fast and accurate short read alignment with Burrows-Wheeler transform. Bioinformatics. 2009;25:1754-60.

44. van de Laar FA, Lucassen PL, Akkermans RP, van de Lisdonk EH, Rutten GE, van Weel C. Alpha-glucosidase inhibitors for patients with type 2 diabetes: results from a Cochrane systematic review and meta-analysis. Diabetes Care. 2005;28:154-63. 
45. Mack M, van Loon AP, Hohmann HP. Regulation of riboflavin biosynthesis in Bacillus subtilis is affected by the activity of the flavokinase/flavin adenine dinucleotide synthetase encoded by ribC. J Bacteriol. 1998;180:950-5.

46. Benson DA, Clark K, Karsch-Mizrachi I, Lipman DJ, Ostell J, Sayers EW. GenBank. Nucleic Acids Res. 2014;42:D32-7.

47. Kanehisa M, Goto S. KEGG: Kyoto Encyclopedia of Genes and Genomes. Nucleic Acids Res. 2000;28:27-30.

48. Omote $\mathrm{H}$, Hiasa M, Matsumoto $T$, Otsuka M, Moriyama $Y$. The MATE proteins as fundamental transporters of metabolic and xenobiotic organic cations. Trends Pharmacol Sci. 2006;27:587-93.

49. Becker ML, Visser LE, van Schaik RH, Hofman A, Uitterlinden AG, Stricker BH. Genetic variation in the multidrug and toxin extrusion 1 transporter protein influences the glucose-lowering effect of metformin in patients with diabetes: a preliminary study. Diabetes. 2009;58:745-9.

50. Tsuda M, Terada T, Mizuno T, Katsura T, Shimakura J, Inui K. Targeted disruption of the multidrug and toxin extrusion 1 (mate1) gene in mice reduces renal secretion of metformin. Mol Pharmacol. 2009;75:1280-6.

51. Kaatz GW, McAleese F, Seo SM. Multidrug resistance in Staphylococcus aureus due to overexpression of a novel multidrug and toxin extrusion (MATE) transport protein. Antimicrob Agents Chemother. 2005;49:1857-64.

52. McAleese F, Petersen P, Ruzin A, Dunman PM, Murphy E, Projan SJ, et al. A novel MATE family efflux pump contributes to the reduced susceptibility of laboratory-derived Staphylococcus aureus mutants to tigecycline. Antimicrob Agents Chemother. 2005;49:1865-71.

53. Hamilton EJ, Martin N, Makepeace A, Sillars BA, Davis WA, Davis TM. Incidence and predictors of hospitalization for bacterial infection in community-based patients with type 2 diabetes: the fremantle diabetes study. PLoS One. 2013;8, e60502.

54. Muller LM, Gorter KJ, Hak E, Goudzwaard WL, Schellevis FG, Hoepelman Al, et al. Increased risk of common infections in patients with type 1 and type 2 diabetes mellitus. Clin Infect Dis. 2005:41:281-8.

55. Qin N, Yang F, Li A, Prifti E, Chen Y, Shao L, et al. Alterations of the human gut microbiome in liver cirrhosis. Nature. 2014;513(7516):59-64.

56. Bloom BH. Space/time trade/offs in hash coding with allowable errors. Communications of the Acm. 1970;13:422.

57. Broder A, Mitzenmacher M. Network applications of bloom filters: a survey. Internet Mathematics. 2004;1:485-509.

58. Rho M, Tang H, Ye Y. FragGeneScan: predicting genes in short and error-prone reads. Nucleic Acids Res. 2010;38, e191.

59. Zhao $Y$, Tang $H$, Ye $Y$. RAPSearch2: a fast and memory-efficient protein similarity search tool for next-generation sequencing data. Bioinformatics. 2012;28:125-6.

60. Overbeek R, Olson R, Pusch GD, Olsen GJ, Davis JJ, Disz T, et al. The SEED and the Rapid Annotation of microbial genomes using Subsystems Technology (RAST). Nucleic Acids Res. 2014;42:D206-14.

61. Strimmer K. fdrtool: a versatile R package for estimating local and tail area-based false discovery rates. Bioinformatics. 2008;24:1461-2.

62. David LA, Maurice CF, Carmody RN, Gootenberg DB, Button JE, Wolfe BE, et al. Diet rapidly and reproducibly alters the human gut microbiome. Nature. 2014:505:559-63.

63. Hingamp P, Grimsley N, Acinas SG, Clerissi C, Subirana L, Poulain J, et al. Exploring nucleo-cytoplasmic large DNA viruses in Tara Oceans microbial metagenomes. ISME J. 2013;7:1678-95.

64. Manges AR, Labbe A, Loo VG, Atherton JK, Behr MA, Masson L, et al. Comparative metagenomic study of alterations to the intestinal microbiota and risk of nosocomial Clostridum difficile-associated disease. J Infect Dis. 2010;202:1877-84

65. Mavromatis K, Ivanova N, Barry K, Shapiro H, Goltsman E, McHardy AC, et al. Use of simulated data sets to evaluate the fidelity of metagenomic processing methods. Nat Methods. 2007;4:495-500.

66. Richter DC, Ott F, Auch AF, Schmid R, Huson DH. MetaSim: a sequencing simulator for genomics and metagenomics. PLoS One. 2008;3, e3373.

\section{Submit your next manuscript to BioMed Central and take full advantage of:}

- Convenient online submission

- Thorough peer review

- No space constraints or color figure charges

- Immediate publication on acceptance

- Inclusion in PubMed, CAS, Scopus and Google Scholar

- Research which is freely available for redistribution

Submit your manuscript at www.biomedcentral.com/submit 